\title{
ON HILBERT CLASS FIELDS IN CHARACTERISTIC $p>0$ AND THEIR $L$-FUNCTIONS
}

\author{
STUART TURNER
}

\begin{abstract}
Let $k$ be a global field of characteristic $p>0$ with field of constants $\mathbf{F}_{q}$. Let $\bar{k}$ be an algebraic closure of $k$. In this note we study the subfields of $\vec{k}$ which are maximal unramified abelian extensions of $k$ with field of constants $\mathbf{F}_{q}$. Each of these fields may be regarded as an analogue of the Hilbert class field of algebraic number theory [1, p. 79]. In $\$ 1$ we recall the construction of these class fields and in $\$ 2$ we show that if $k$ has genus one, they are all $\mathbf{F}_{q}$-isomorphic. In $\$ 3$ we show that this is not necessarily the case if the genus of $k$ is greater than one. The argument there is based on an observation about the $L$-functions of the fields.
\end{abstract}

1. Let $k_{A}^{x}$ be the idele group of $k$ and identify $k^{x}$ with the principal ideles in $k_{A}^{x}$. Let $k_{A}^{1}$ be the ideles of module 1 and $U$ be the maximal compact subgroup of $k_{A}^{x}$. Let $D$ be a complete nonsingular curve defined over $\mathbf{F}_{q}$ with function field isomorphic to $k . D$ is unique up to $\mathbf{F}_{q}$ isomorphism. Let $J(D)\left(\mathbf{F}_{q}\right)$ denote the group of $\mathbf{F}_{q}$-rational points on $J(D)$, the Jacobian variety of $D . J(D)\left(\mathbf{F}_{q}\right)$ is a finite group. Let $h=\operatorname{card}\left(J(D)\left(\mathbf{F}_{q}\right)\right) \cdot k_{A}^{1} / k^{x} U$ is canonically isomorphic to $J(D)\left(\mathbf{F}_{q}\right)$.

We now recall straightforward (and well-known) consequences of the existence theorem [1, Chapter VIII, in particular §3], [3, Chapter XIII, §9].

Let $z \in k_{A}^{x}$ with module $(z)=q$. Let $u_{1}, \ldots, u_{h}$ be representatives of the cosets of $k^{x} U$ in $k_{A}^{1}, u_{1} \in k^{x} U$. Then $N_{i}=\left\{z u_{i}\right\} \times k^{x} U$ are distinct open subgroups of $k_{A}^{x}$ and each $k_{A}^{x} / N_{i}$ is canonically isomorphic to $k_{A}^{1} / k^{x} U$. The class fields $k_{1}, k_{2}, \ldots, k_{h}$ of $N_{1}, N_{2}, \ldots, N_{h}$, respectively, are unramified abelian extensions of $k$ each with constant field $\mathbf{F}_{q}$ and each $\operatorname{Gal}\left(k_{i} / k\right)$ is canonically isomorphic to $J(D)\left(\mathbf{F}_{q}\right)$.

Furthermore, these $k_{i}$ are the only maximal unramified abelian extensions of $k$ with constant field $\mathbf{F}_{q}$ because any $x \in k_{A}^{x}$ with module $(x)=q$ lies in one of the cosets $z u_{i} k^{x} U$ of $k^{x} U$ in $k_{A}^{x}$.

Let $L$ be the constant field extension of $k$ of degree $h . L$ is the class field of the subgroup $\left\{z^{h}\right\} \times k_{A}^{1}$ so $L k_{i}$ is the class field of

$$
\left\{z^{h}\right\} \times k_{A}^{1} \cap\left\{z u_{i}\right\} \times k^{x} U=\left\{\left(z u_{i}\right)^{h}\right\} \times k^{x} U, \quad i=1, \ldots, h .
$$

But $\left(z u_{i}\right)^{h}$ and $\left(z u_{j}\right)^{h}$ represent the same coset of $k_{A}^{1}$ in $k_{A}^{x}$, so $L k_{i}=L k_{j}$, $1 \leqslant i, j \leqslant h$.

Let $C_{i}$ be a complete nonsingular curve defined over $\mathbf{F}_{q}$ with function field

Received by the editors March 8, 1976.

AMS (MOS) subject classifications (1970). Primary 12A65, 14G10, 14H30; Secondary 12A90, $14 \mathrm{H} 40$. 
isomorphic to $k_{i}$. The $C_{i}$ are unique up to $\mathbf{F}_{q}$-isomorphism. Since $k_{i}$ is an unramified extension of $k$, there exist surjective étale morphisms $\gamma_{i}: C_{i} \rightarrow D$ defined over $\mathbf{F}_{q}$. Let $g$ be the genus of $D$; then, $g\left(C_{i}\right)$, the genus of $C_{i}$, is given by $2 g\left(C_{i}\right)-2=h(2 g-2)$ [3, Chapter VIII, Corollary to Proposition 14]. Summarizing the discussion in geometric terms we have

THEOREM 1. Let $D$ be a complete nonsingular curve of genus $g$ defined over $\mathbf{F}_{q}$. Let $J(D)$ be the Jacobian variety of $D$ and $G=J(D)\left(\mathbf{F}_{q}\right)$ be the group of $\mathrm{F}_{q}$-rational points of $J(D)$. Let $h=$ card $G$. Then there exist $h$ complete nonsingular curves $C_{i}$ defined over $\mathbf{F}_{q}$ each of genus $h(g-1)+1$, and morphisms $\gamma_{i}: C_{i} \rightarrow D$ defined over $\mathbf{F}_{q}$ such that $\gamma_{i}$ is an étale cover of degree $h$. The Galois group of the cover $\gamma_{i}$ is isomorphic to $G$.

Observe that for $i \neq j$ there does not exist any morphism $\delta: C_{i} \rightarrow C_{j}$ such that $\gamma_{j} \circ \delta=\gamma_{i}$; for the existence of such a morphism would imply the existence of a $k$-isomorphism of $k_{j}$ onto a subfield of $k_{i}$, but this is impossible because $k_{j}$ and $k_{i}$ are distinct normal extensions of $k$ in $\bar{k}$.

However, if $D$ has genus one, $C_{i}$ is $\mathbf{F}_{q}$-isomorphic to $C_{j}$ for all $i, j, 1 \leqslant i$, $j \leqslant h$. This is proven in $\S 2$.

\section{2.}

Lemma 1. Let $v$ be a place of $k$ of degree one. Then $v$ splits completely in precisely one of the class fields $k_{i}, 1 \leqslant i \leqslant h$.

Proof. The places of $k_{i}$ which lie above $v$ are in one-to-one correspondence with the cosets of $k_{v}^{x} N_{i}$ in $k_{A}^{x}$ [3, Chapter XIII, Proposition 14], so $v$ splits completely in $k_{i}$ if and only if $\left[k_{A}^{x}: k_{v}^{x} N_{i}\right]=h$. On the other hand, $\left[k_{A}^{x}\right.$ : $\left.N_{i}\right]=h$, so $v$ splits completely in $k_{i}$ if and only if $k_{v}^{x} \subset N_{i}=\left\{z u_{i}\right\} \times k^{x} U$. Let $r_{v}$ be the valuation ring in $k_{v} \cdot r_{v}^{x} \subset N_{i}$ for all $i, 1 \leqslant i \leqslant h$. Let $\pi_{v}$ be a prime element in $r_{v}$. Since $v$ is a place of degree one, module $\left(\pi_{v}^{-1}\right)=q$. So $\pi_{v} \in N_{i}$ if and only if $\pi_{v} z^{-1} \in u_{i} k^{x} U$; there is a unique $i$ for which this is the case.

REMARK. The hypothesis of Lemma 1 is not always satisfied. There exist global fields which do not have places of degree one.

LEMMA 2. If $k$ has genus one and $k_{i}$ is the class field determined by $N_{i}$, then there is a unique place $v$ of $k$ of degree one that splits completely in $k_{i}$.

Proof. $k_{i}$ has genus one, hence has a place $w$ of degree one. $w$ has residue field $\mathbf{F}_{q}$ and lies over a place $v$ of $k$ of degree one. $w$ has $h$ distinct conjugates $w=w_{1}, \ldots, w_{h}$ over $v$ because $\sum e\left(w_{i}\right) f\left(w_{i}\right)=h, e\left(w_{i}\right)=1$ for $i=1, \ldots, h$, and $f(w)=1$.

THEOREM 2. Let notations be as in Theorem 1 and assume that $D$ is a curve of genus one. Then there is a canonical one-to-one correspondence between the rational points of $D$ and the curves $C_{i}$. A rational point $P$ of $D$ corresponds to the curve $C_{i}$ if and only if there are $h$ points of $C_{i}$ in the fiber $\gamma_{i}^{-1}(P)$. 
Proof. $D$ is $\mathbf{F}_{q}$-isomorphic to $J(D)$, so $D$ has $h$ rational points. The theorem now follows from Lemmas 1 and 2.

Throughout the rest of this section we assume that $k$ has genus one.

Let $v$ be a place of $k$ and $\rho: k \rightarrow k_{v}$ be an embedding of $k$ into the completion of $k$ at $v$. Let $k^{\prime}$ be a field and $\alpha: k^{\prime} \rightarrow k$ be an isomorphism. Denote by $\alpha v$ the place of $k^{\prime}$ arising from the embedding $\rho \circ \alpha: k^{\prime} \rightarrow k_{v}$. Denote by $v(i)$ the place of $k$ which corresponds to the class field $k_{i}$.

LEMMA 3. Let $\beta: k_{j} \rightarrow k_{i}$ be an $\mathbf{F}_{q}$-isomorphism such that $\beta(k) \subset k$ and let $\alpha=\left.\beta\right|_{k}$. Then $v(j)=\alpha v(i)$. Conversely, if $\alpha: k \rightarrow k$ is an $\mathbf{F}_{q}$-isomorphism such that $v(j)=\alpha v(i)$, then there is an $\mathbf{F}_{q}$-isomorphism $\beta: k_{j} \rightarrow k_{i}$ such that $\left.\beta\right|_{k}=$ $\alpha$.

Proof. Let $w$ be a place of $k_{i}$ of degree one. $\beta w$ is a place of $k_{j}$ of degree one. By Lemma $2, w$ lies over $v(i)$ and $\beta w$ lies over $v(j)$ so $v(j)=\alpha v(i)$.

To prove the converse observe that there are $h$ distinct embeddings $\beta_{i}$ : $k_{j} \rightarrow \bar{k}, 1 \leqslant i \leqslant h$, such that $\left.\beta_{i}\right|_{k}=\alpha$.

The $\beta_{i}$ all have the same image $L$ in $\bar{k} . L$ is an unramified abelian extension of $k$ with field of constants $\mathbf{F}_{q}$. It suffices to show that $L=k_{i}$. Let $w$ be a place of $k_{j}$ of degree one and $u$ be the place of $\beta_{1}\left(k_{j}\right)$ such that $w=\beta_{1} u$. By Lemma $2, w$ lies over $v(j)$ and $u$ lies over $v(i)$ because $v(j)=\alpha v(i)$. So $u$ is a place of $k_{i}$ and $L=k_{i}$.

Let $k(D), k\left(C_{i}\right)$ and $k\left(C_{j}\right)$ be the function fields of $D, C_{i}$ and $C_{j}$, respectively. The morphisms $\gamma_{i}$ and $\gamma_{j}$ of Theorem 1 define injections $\gamma_{i}^{*}$ : $k(D) \rightarrow k\left(C_{i}\right)$ and $\gamma_{j}^{*}: k(D) \rightarrow k\left(C_{j}\right)$. Choose $\mathbf{F}_{q}$-isomorphisms of $k(D)$ with $k$, of $k\left(C_{i}\right)$ with $k_{i}$, and of $k\left(C_{j}\right)$ with $k_{j}$; so that $\gamma_{i}{ }^{*}$ (resp. $\gamma^{*}$ ) is compatible with the inclusion $k \subset k_{i}$ (resp. $k \subset k_{j}$ ). Identify $k(D)$ with $k, k\left(C_{i}\right)$ with $k_{i}$, and $k\left(C_{j}\right)$ with $k_{j}$ by means of these isomorphisms. The places $v(i), 1 \leqslant i \leqslant$ $h$, of $k$ are thus identified with places of $k(D)$. Let $P_{i}, 1 \leqslant i \leqslant h$, be the rational points of $D$ corresponding to the places $v(i), 1 \leqslant i \leqslant h$, of $k(D)$, respectively.

THEOREM 3. Let the notations be as in Theorem 1 and assume that $D$ is a curve of genus one. Let $\eta$ be an $\mathbf{F}_{q}$-automorphism of $D$. Then there exists an $\mathbf{F}_{q}$-isomorphism $\delta: C_{i} \rightarrow C_{j}$ such that $\gamma_{j} \circ \delta=\eta \circ \gamma_{i}$ if and only if $\eta\left(P_{i}\right)=P_{j}$.

Proof. Let $\eta^{*}: k(D) \rightarrow k(D)$ be the automorphism of $k(D)$ induced by $\eta$. $\eta\left(P_{i}\right)=P_{j}$ is equivalent to the condition $v(j)=\eta^{*} v(i)$. By Lemma 3 there is an $\mathbf{F}_{q}$-isomorphism $\beta: k\left(C_{j}\right) \rightarrow k\left(C_{i}\right)$ such that $\left.\beta\right|_{k(D)}=\eta^{*} . \beta$ determines an $\mathbf{F}_{q}$-isomorphism $\delta: C_{i} \rightarrow C_{j}$ such that $\gamma_{j} \circ \delta=\eta \circ \gamma_{i}$. The proof of the converse follows similarly from the first assertion of Lemma 3.

COROllary. Let the notations be as in Theorem 1 and assume that $D$ has genus one. Then there exist $\mathbf{F}_{q}$-isomorphisms $\delta: C_{i} \rightarrow C_{j}$ for all $i, j, 1 \leqslant i, j \leqslant h$.

Proof. Since $D$ has genus one, the group of $\mathbf{F}_{q}$-isomorphisms of $D$ acts transitively on the $\mathbf{F}_{q}$-rational points of $D$. The assertion now follows from the theorem. 
3. Returning to the discussion in $\S 1$, recall that the fields $k_{i} \subset \bar{k}$ were defined as the class fields of subgroups $N_{i}$ of $k_{A}^{x}$. Let $\Omega_{i}$ be the group of characters of $k_{A}^{x}$ trivial on $N_{i}$ and $\Omega_{i}^{\prime}$ be the elements of $\Omega_{i}$ distinct from the trivial character. Then the Dedekind zeta function of $k_{i}$ is given by $\zeta_{k_{i}}(s)=$ $\zeta_{k}(s) \cdot \Pi_{\omega \in \Omega_{i}^{\prime}} L(s, \omega)[3$, Chapter XIII, §10].

In case $k$ has genus one, the $L(s, \omega)$ are all identically one [3, Chapter VII, $\S 7$, but if the genus of $k$ is greater than one, these $L$-functions are nontrivial. Throughout this section we assume that the genus of $k$ is at least two.

For $s \in \mathbf{C}$, let $\omega_{s}: k_{A}^{x} \rightarrow \mathbf{C}^{x}$ be the quasicharacter defined by $\omega_{s}(z)=|z|^{s}$; $\omega_{s}: k_{A}^{1} \rightarrow 1$.

LEMMA 4. Let $\omega \in \Omega_{1}, \omega \neq 1$, and let $\omega\left(z u_{i}\right)=q^{-s_{i}(\omega)}$. There is a one-to-one correspondence between $\Omega_{1}$ and $\Omega_{i}$ given by $\omega \leftrightarrow \omega \omega_{s_{i}(\omega)}$.

Proof. $\omega$ has order $h$ so $q^{-s_{i}(\omega)}$ is an $h$ th root of one and $s_{i}(\omega)$ is defined modulo elements of $(2 \pi i / \log q) \mathbf{Z} . \omega$ and $\omega_{s}$ induce the trivial character on $k^{x} U$, so $\omega \omega_{s} \in \Omega_{i}$ if and only if $\omega \omega_{s}\left(z u_{i}\right)=1$.

This is equivalent to $s \equiv s_{i}(\omega)(\bmod (2 \pi i / \log q) \mathbf{Z})$. The verification that the correspondence between $\Omega_{1}$ and $\Omega_{i}$ is one-to-one is left to the reader.

Lemma 4 and the definition of the $L$-functions give

Proposition.

$$
\zeta_{k_{i}}(s)=\zeta_{k}(s) \prod_{\omega \in \Omega_{1}^{\prime}} L\left(\omega \omega_{s_{i}(\omega)}, s\right)=\zeta_{k}(s) \prod_{\omega \in \Omega_{1}^{\prime}} L\left(\omega, s+s_{i}(\omega)\right) .
$$

$\zeta_{k_{i}}=\zeta_{k_{j}}$, for $1 \leqslant i, j \leqslant h$, if and only if $J\left(C_{i}\right)$ is $\mathbf{F}_{q}$-isogenous to $J\left(C_{j}\right)$ [2, Theorem 1].

COROllary. Let the notations be as in Theorem 1 and assume that $D$ has genus at least two and that $h=2$. Then $J\left(C_{1}\right)$ is not $\mathbf{F}_{q}$-isogenous to $J\left(C_{2}\right)$ and, hence, $C_{1}$ is not $\mathbf{F}_{q}$-isomorphic to $C_{2}$.

Proof. Let $\omega \in \Omega_{1}^{\prime}$; then $\zeta_{k_{1}}(s)=\zeta_{k_{2}}(s)$ if and only if

$$
L(\omega, s)=L\left(\omega, s+s_{2}(\omega)\right) \text {, }
$$

where $q^{-s_{2}(\omega)}=-1$ because $\omega \omega_{s_{2}(\omega)} \in \Omega_{2}^{\prime}$. So

$$
s_{2}(\omega) \equiv \pi i / \log q(\bmod (2 \pi i / \log q) \mathbf{Z}) .
$$

On the other hand $L(\omega, s)$ has period $2 \pi i / \log q$, so

$$
L(\omega, s) \neq L\left(\omega, s+s_{2}(\omega)\right) \text {. }
$$

\section{REFERENCES}

1. E. Artin and J. Tate, Class field theory, Benjamin, New York, 1968. MR 36 \#6383.

2. J. Tate, Endomorphisms of abelian varieties over finite fields, Invent. Math. 2 (1966), 134-144. MR 34 \# 5829.

3. A. Weil, Basic number theory, Grundlehren math. Wiss. Band 144, Springer-Verlag, New York, 1967. MR 38 \#3244.

Departamento de Matemática, Pontificia Universidade Católica do Rio de Janeiro, Rua Marquês de São Vicente 209/263, Rio de JaNeiro, Brazil 